\title{
La implementación de las recomendaciones de la auditoría del Sistema Nacional de Control y la gestión de los gobiernos locales de Lima Metropolitana, periodo 2015-2020
}

\section{Martín Edmundo Mogrovejo Espinoza}

Escuela de Auditoría, Facultad de Ciencias Contables Universidad Nacional Mayor de San Marcos

El presente estudio tiene como objetivo general determinar en qué medida el incumplimiento de la implementación de las recomendaciones de la auditoría del Sistema Nacional de Control (SNC) afecta la gestión de los gobiernos locales de Lima Metropolitana en el periodo 2015-2020. El tipo de estudio es básico; el nivel de la investigación es descriptivo-explicativo, y el diseño es no experimental, de corte transversal, transeccional y correlacional-causal. La población de estudio está dada por las 43 municipalidades distritales de la provincia de Lima y el tamaño de la muestra corresponde a 12 municipalidades distritales. Las técnicas para la recopilación de datos fueron el análisis documental y la entrevista, mientras que el instrumento fue el cuestionario. Sobre la base de los resultados obtenidos, la variable implementación de las recomendaciones de la auditoría orientadas a mejorar la gestión de la entidad alcanzaron una tendencia media de aceptación en la gestión de las municipalidades en cuanto a la capacidad del manejo de los recursos, eficiencia y procedimientos. Por otro lado, en la variable gestión de los gobiernos locales, la eficiencia ha sido considerada medianamente aceptable. Así, se garantiza parcialmente el cumplimiento de tareas, la productividad y el consumo de materiales. Entre las conclusiones, se resalta la eficiencia por el cumplimiento regular de las tareas de los gobiernos locales; además, estas han sido valoradas de forma regular, principalmente, por la poca adecuación a las recomendaciones señaladas por el SNC.

Palabras clave: auditoría, recomendaciones, gestión de gobiernos locales

\section{The implementation of the recommendations of the audit of the National Control System and the manage- ment of the local governments of Lima Metropolitana, period 2015-2020}

The general objective of this study is to determine to what extent non-compliance with the implementation of the recommendations of the audit of the National Control System (SNC) affects the management of the local governments of Metropolitan Lima in the 2015-2020 period. The type of study is basic; the research level is descriptive-explanatory and the design is non-experimental, cross-sectional, transectional and correlational-causal. The study population is given by the 43 district municipalities of the province of Lima and the sample size corresponds to 12 district municipalities. The techniques for data collection were the documentary analysis and the interview, while the instrument was the questionnaire. Based on the results obtained, the variable Implementation of the audit recommendations aimed at improving the management of the entity reached an average acceptance trend in the management of the municipalities in terms of: resource management capacity, efficiency and procedures. On the other hand, the variable local government management efficiency has been considered moderately acceptable; Thus, the fulfillment of tasks, productivity and the consumption of materials are partially guaranteed. Among the conclusions, the efficiency for the regular fulfillment of the tasks of the local governments stands out; Furthermore, these have been regularly assessed, mainly due to the lack of adaptation to the recommendations indicated by the SNC.

Keywords: audit, recommendations, local government management 


\section{A implementaçáo das recomendaçóes da auditoria do Sistema De Controlo Nacional e a gestáo dos governos locais da Lima Metropolitana, período 2015-2020}

O objetivo geral deste estudo é determinar em que medida o não cumprimento da implementaçấo das recomendações da auditoria do Sistema de Controle Nacional (SNC) afeta a gestão das prefeituras da região metropolitana de Lima no período 2015-2020. O tipo de estudo é básico; o nível de pesquisa é descritivo-explicativo e o delineamento é não experimental, transversal, transversal e correlacional-causal. A população do estudo é dada pelos 43 municípios distritais da província de Lima e o tamanho da amostra corresponde a 12 municípios distritais. As técnicas de coleta de dados foram a análise documental e a entrevista, enquanto o instrumento foi o questionário. Com base nos resultados obtidos, a variável Implementaçáo das recomendaçôes da auditoria com vista à melhoria da gestão da entidade atingiu uma tendência média de aceitação na gestão dos municípios em termos de: capacidade de gestão de recursos, eficiência e procedimentos. Por outro lado, a variável eficiência da gestáo do governo local foi considerada moderadamente aceitável; Assim, o cumprimento das tarefas, a produtividade e o consumo de materiais ficam parcialmente garantidos. Dentre as conclusões, destaca-se a eficiência para o cumprimento regular das tarefas dos governos locais; Além disso, estes têm sido avaliados regularmente, principalmente devido à falta de adaptação às recomendações apontadas pelo SNC.

Palavras-chave: auditoria, recomendações, administração do governo local

\section{Introducción}

La auditoría tiene un papel importante para asegurar el logro de los objetivos de las organizaciones, pues tiene como función principal proporcionar las garantías necesarias. Más allá de la evaluación y la contribución a la mejora del gobierno corporativo, la auditoría sirve para garantizar la gestión de riesgos, y los procesos de control interno guiados por enfoques sistemáticos y disciplinados (Wang, Liu, \& Chang, 2011). En ese sentido, el control gubernamental tiene un impacto en la auditoria, que tiene por finalidad ayudar a una organización a cumplir sus objetivos aportando un enfoque sistemático y disciplinado para evaluar $\mathrm{y}$ mejorar la efectividad de los procesos de gestión de riesgos, control y dirección.

Toda auditoría elabora un informe que consta de cuatro partes básicas: (i) aspectos preliminares; (ii) características de la entidad, programa, proyecto, planes, etc.; (iii) resultados de la auditoría; y (iv) disposiciones finales, que, a su vez, están compuestas por las con- clusiones y recomendaciones. Las recomendaciones son las propuestas de acción dirigidas a la autoridad competente con el fin de corregir los errores, omisiones y desviaciones determinadas (Instituto de Altos Estudios de Control Fiscal y Auditoría de Estado [COFAE], 2014, p.7).

En tal sentido, las recomendaciones son tareas para la corrección y provisión que se exhiben en los informes de un proceso de auditoría. Son el resultado de las decadencias o informalidades derivadas de un proceso de inspección y son direccionadas a los encargados competentes que tienen el compromiso de llevarlas a cabo. Dichas acciones son propuestas por el Sistema Nacional de Control (en adelante, SNC). Luego de ser notificadas, las recomendaciones son de implementación forzosa bajo el control de la Contraloría General de la República. Los Órganos de Control Institucional (en adelante, OCI) se encargan el seguimiento de las medidas correctivas. Las recomendaciones no son potestativas o discrecionales, sino de carácter impe- 
rativo; es decir, para el funcionario u autoridad edil son ineludibles: son disposiciones de cumplimiento obligatorio.

Las Normas Generales de Control Gubernamental establecen desde los numerales 4.17 al 4.19 el seguimiento a la implementación de las recomendaciones. Por lo tanto, la Contraloría y los OCI deben realizar el seguimiento de las acciones que las entidades dispongan para la implementación efectiva y oportuna de las recomendaciones formuladas en los resultados de los servicios de control. Estos pueden contener recomendaciones, que son entendidas como medidas concretas y posibles dirigidas a mejorar la eficiencia de la gestión de las entidades en el manejo de sus recursos y en los procedimientos que emplean en su accionar. Además, por medio de las recomendaciones, se busca contribuir a la transparencia de la gestión de las entidades.

El SNC efectúa las auditorías en las entidades del Estado. Entre ellas, se encuentran los gobiernos locales, que tienen como misión principal el servicio social, como se observa en las obras en sus planes operativos. Entre los gobiernos locales, se encuentran las municipalidades provinciales y distritales, que son organismos públicos con personería jurídica de derecho público (Ley 27972, 2003, art. 1) y con sujeción al ordenamiento jurídico (art. 2). Por ende, las municipalidades están sujetas a la fiscalización y control que se realiza a través de los OCI, que realizan auditorías internas y emiten informes con las recomendaciones que la municipalidad correspondiente debe ejecutar para subsanar las deficiencias detectadas.

Cabe señalar que, en enero de 2018, Lima Metropolitana tenía una población de 9 millones 320 mil habitantes (Instituto Nacional de Estadística e Informática [INEI], 2018). Hasta la actualidad, la ciudad es gestionada por 43 gobiernos locales distritales. La importancia de Lima estriba en que es la capital del país, y el centro político, cultural, financiero y comercial. Para el año fiscal de 2018, a los gobiernos locales de la ciudad se les asignó S/ 16.025.792.206 del presupuesto anual de gastos para gastos corrientes, gastos de capital y servicio de la deuda. Concretamente, a la provincia de Lima se le asignó un presupuesto de S/ 4.752.162.620 (Ministerio de Economía y Finanzas [MEF], 2015), que corresponde a 29,7\% de lo asignado a los gobiernos locales del país. Entonces, la cifra denota la importancia de Lima como unidad política-administrativa del país. De dicho, presupuesto, se consideran gastos de bienes, servicios y adquisición de activos a través de programas y pliegos presupuestales que, tras su ejecución, son objeto de auditorías llevadas a cabo por la Contraloría General de la República.

Para fines del presente estudio, se ha optado por seleccionar municipalidades distritales por su importancia social (densidad población), económica o política $^{2}$, su representatividad, la ejecución de proyectos, planes, programas para atender a su población que demanda servicios, y obras. Como se ha mencionado, la provincia de Lima está constituida por 43 municipalidades distritales, entre los que destacan la Municipalidad Metropolitana de Lima, que es la municipalidad con mayor presupuesto asignado en el país (S/ 4.752.162.620). Además, por ser la municipalidad de la capital del país, tiene un peso significativo a nivel político, cultural, social y económico. Por otro lado, los distritos de San Juan de Lurigancho

\footnotetext{
2 Al ser la municipalidad de la capital del país, la Municipalidad Metropolitana de Lima (MML) tiene un peso político gravitante sobre el
} resto de las municipalidades. 
(el distrito más poblado del país con 1.091.303 habitantes), San Martín de Porres (700.178 habitantes), Ate (630.085), Comas (524.894) y Villa El Salvador (463.014) son los distritos más poblados de Lima; también se constituyen como poblados pujantes, y preocupados por mejorar su situación económica, cultural y social. Esto demanda la ejecución de obras y servicios. También se encuentran los distritos tradicionales, como Breña, Chorrillos y Jesús María, que muestran un desarrollo urbanístico horizontal y comercial. A estos distritos se les suma San Isidro, que es considerado el corazón financiero de la capital y el distrito de mayor crecimiento urbanístico. Entonces, al seleccionar los gobiernos locales de Lima, se configura una muestra representativa de las diversas realidades sociales, económicas, culturales y urbanísticas gestionadas con diversas políticas de sus gobiernos locales.

En el periodo 2015-2020, en las municipalidades se registraron 1.985 recomendaciones en los informes de auditorías realizadas. De ellas, solo 891 recomendaciones se han implementado, es decir, el 44,89\% del total. Esto evidencia que más de la mitad de las recomendaciones no se han implementado. Se destacan algunos factores sobre incumplimiento, como la desidia, el desinterés, la falta de claridad en las recomendaciones y la inexistencia de una penalidad por la falta, entre otros.

La implementación de las recomendaciones tiene por objetivo corregir o mejorar aspectos de la gestión edil. Por ello, cuando una recomendación no es implementada, la población y la misma institución se ven afectadas. Así, cuando en un informe de auditoría sobre las municipalidades de San Miguel, San Martín de Porres, Comas y Pachacamac se recomienda mejorar el sistema de rendición de cuentas, y dicha recomendación no se toma oportunamente, habrá fallas en el cumplimiento de los requisitos y las formalidades establecidas en la normativa vigente y aplicable a los procedimientos administrativos y operativos asociados al pago de obligaciones por adquisiciones de bienes y servicios a fin de cautelar su debido sustento técnico y legal. De no aplicar las recomendaciones, los directivos y encargados de gestionar tales actividades tendrían que asumir las responsabilidades administrativas y penales por este incumplimiento.

De tal modo, la determinación de las responsabilidades administrativas y penales aplicables a los funcionarios o servidores públicos tiene un fin reparador y sancionador según los delitos comunes incurridos (Ley 27785, 2018, art. 6). En cambio, si se implementa la recomendación, se adoptarían acciones para detectar y corregir errores e irregularidades a través de un sistema integrado, que asegure la confiabilidad, calidad, suficiencia y oportunidad de la información vinculada al régimen fiscalizador y sancionador. Además, mejoraría la imagen institucional de la municipalidad, y la gestión del alcalde se vería reforzada y compensada por el reconocimiento de la población. En este sentido, es necesario identificar por qué las municipalidades distritales no implementan las recomendaciones de las auditorías. Asimismo, hay que indagar de qué manera este incumplimiento afecta la gestión de las municipalidades distritales de Lima Metropolitana.

A partir de las ideas comentadas, se formula el problema de la presente propuesta de investigación con la siguiente interrogante: ¡en qué medida el incumplimiento de la implementación de las recomendaciones de la auditoría del SNC afecta la gestión de los gobiernos locales de Lima Metropolitana en el periodo 2015-2020? A partir de ello, el objetivo general de la investigación consiste en determinar en qué medida el incumplimiento de la implementación de las reco- 
mendaciones de la auditoría del SNC afecta la gestión de los gobiernos locales de Lima Metropolitana en el periodo 2015-2020.

Sobre la base de la pregunta y el objetivo de la investigación, se comentarán los antecedentes del estudio. En primer lugar, se encuentra la investigación de Ramos (2017), que plantea como propósito determinar el nivel de cumplimiento en la implementación de las recomendaciones planteadas en los informes de auditoría emitidos por el OCI y su incidencia en la gestión del Gobierno Regional de Puno en los periodos de 2013-2014. Así, concluye que la adecuación de las recomendaciones señaladas en los dictámenes de auditoría formuladas por el OCI incide de forma negativa en la dirección del Gobierno Regional Puno en los periodos de 2013 - 2014. Esto se explica a partir de razones como la ausencia de cierto mecanismo de regulación de la institución.

En segundo lugar, Medrano (2017) analiza si la evaluación de deficiencias del informe de auditoría financiera influye en el mejoramiento de la gestión de los gobiernos locales de Lima Metropolitana en el periodo 2013-2014. A partir de ello, concluye que el alcance de la ejecución de las adecuaciones afecta la eficacia de la dirección de las administraciones locales.

En tercer lugar, Gaibor (2015) realizó un estudio que tuvo como propósito evaluar el grado de cumplimiento de recomendaciones de auditoría y su influencia en los resultados de los procesos administrativos y financieros del Gobierno Autónomo Descentralizado Municipal del cantón Montalvo de la provincia los Ríos. Entre las conclusiones, destaca que el caso analizado refleja un bajo nivel de aplicación de las reglas de control interno, de forma previa, corriente y posterior. Esto genera fallas en los procedimientos de administración de la institución.
En cuarto lugar, la investigación de Molina (2018) tuvo como finalidad analizar la implementación de recomendaciones de los informes de auditoría de cumplimiento que permite la adecuada gestión administrativa en la Municipalidad Distrital de Acoria, Huancavelica en el periodo 2015-2016. Entre los hallazgos, se señala que una proporción mínima de las labores de control ejecutadas por el titular ha ocasionado una inoportuna gerencia administrativa, así como inconvenientes en los seguimientos de tareas correccionales por parte del OCI. Esto se debe a que en la mayor parte de los asuntos no se consigue dar objeción a las solicitudes para la comprobación de las acciones realizadas por los responsables.

Por último, Apaico (2019) estudia cómo la implementación de recomendaciones de los informes de auditoría gubernamentales incide en la gestión administrativa de la Municipalidad Provincial de Huamanga en 2018. Entre las conclusiones, se determina que el OCI y el titular de la Municipalidad Provincial de Huamanga realizaron la ejecución y seguimiento de los señalamientos de los dictámenes de auditoría con la finalidad de mejorar y fortalecer los procedimientos administrativos en beneficio de la gestión administrativa de la entidad. Así, se evidencia que han cumplido con las regulaciones legales formuladas por la Contraloría General de la República.

Sobre la base de las investigaciones revisadas, surge la siguiente hipótesis general del presente estudio: el incumplimiento de la implementación de las recomendaciones de la auditoría del SNC afecta significativamente la gestión de los gobiernos locales de Lima Metropolitana en el periodo 2015-2020. La investigación se justifica desde el punto de vista teórico a partir de la teoría de la gestión, la teoría de la gestión municipal y, el marco legal municipal, así como por la teoría sobre administración, economía, planeamiento

Contabilidad y Negocios (16) 31, 2021 / e-ISSN 2221-724X 
y auditoría. Estas bases concurrirán para fundamentar y evaluar técnicamente la propuesta de solución. Desde el punto de vista práctico se estableció la relación entre las inversiones y los programas sociales con la no implementación de las recomendaciones de las auditorías en las municipalidades distritales de Lima Metropolitana. Con respecto a la metodología para lograr los objetivos de la investigación, se realizó el análisis unidimensional de los resultados del trabajo de campo, es decir, al análisis de la data glosa conforme a los objetivos e hipótesis sobre la ejecución de las sugerencias de los dictámenes de auditoría con respecto a la gestión de los gobiernos locales de Lima Metropolitana del periodo 2015-2020.

\section{Material y métodos}

El presente estudio es una investigación de tipo básica, porque permite reconocer las diferentes teorías científicas existentes en relación al problema de estudio. El nivel o alcance de la investigación es descriptivoexplicativo, de manera que en este estudio se busca determinar la relación existente entre la implementación de las recomendaciones de la auditoría del SNC y la gestión de los gobiernos locales distritales de la provincia de Lima. El diseño de la investigación es no experimental, de corte transversal, y transeccional correlacional-causal, puesto que el estudio está orientado a evaluar la relación existente entre los resultados de la implementación de las recomendaciones de la auditoría y la gestión de los gobiernos locales distritales de la provincia de Lima.

La población de estudio corresponde a las 43 municipales distritales de la provincia de Lima, a las que se les han realizado auditoría entre los años 2015 y 2020, y se les han emitido recomendaciones que imperativamente se han debido implementar. Así mismo, a partir de la técnica de muestra opinática o intencional, se tomará como tamaño de muestra a 12 municipalidades distritales de Lima Metropolitana. En este sentido, se confeccionó una muestra propositiva y de carácter opinático a los encargados del control (monitoreo) de la implementación de las recomendaciones, que se estima que es de 12 personas. La selección de la muestra se realizó de manera no probabilística y por conveniencia, dado que no dependerá de la probabilidad sino de las características primordiales de los sujetos de estudio: se selecciona la municipalidad en la que el titular facilitó el acceso a la información y el monitoreo de la aplicación de las recomendaciones.

Por otro lado, las técnicas para la recopilación de datos (trabajo de campo) fueron las siguientes: la técnica del análisis documental, que consiste en revisar libros, revistas especializadas y documentos relacionados al tema del estudio; y la técnica de encuesta, para la que se utiliza el cuestionario como instrumento.

En el desarrollo de la investigación, se aplicó el instrumento del cuestionario a los responsables de la aplicación de las recomendaciones en las municipalidades distritales de la provincia de Lima. El cuestionario constó de una batería de 30 preguntas, de las cuales 15 corresponden a la variable independiente (implementación de las recomendaciones de la auditoría); y 15 preguntas, a la variable dependiente (gestión de gobiernos locales).

\section{Resultados}

En relación con la pregunta “ ¿Ha sido favorable la capacidad del manejo de los recursos una vez implantadas las recomendaciones orientadas a mejorar la gestión de la municipalidad?", 66,67\% de los encuestados (es decir, la mayoría de ellos) contestaron "algunas veces"; 16,67\% "casi nunca"; 8,33\% "casi siempre"; y $8,33 \%$, "nunca" (ver figura 1 ). 


\section{Figura 1. Gráfico de distribución de frecuencia - pregunta 1}

1. ¿Ha sido favorable la capacidad del manejo de los recursos una vez implantadas las recomendaciones orientadas a mejorar la gestión de la municipalidad?

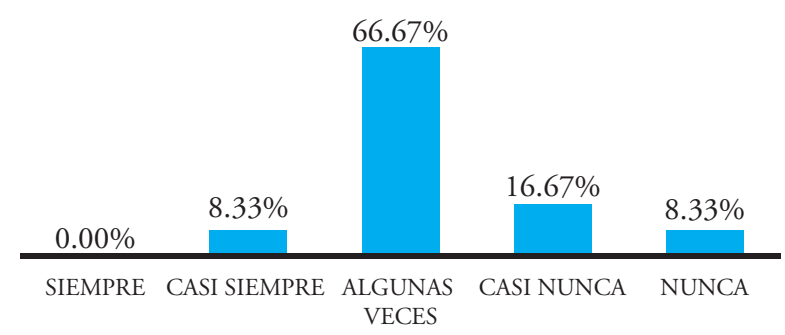

Con respecto a la pregunta “ ${ }_{\complement} \mathrm{Ha}$ decaído el nivel de eficiencia en el manejo de los recursos por el incumplimiento de las recomendaciones orientadas a la gestión de la municipalidad?", $50 \%$ de los encuestados contestaron "algunas veces"; 25\% "casi siempre"; y $25 \%$, "casi nunca" (ver figura 2).

\section{Figura 2. Gráfico de distribución de frecuencia - pregunta 2}

2. ¿Ha decaído el nivel de eficiencia en el manejo de los recursos por el incumplimiento de las recomendaciones orientadas a la gestión de la municipalidad?

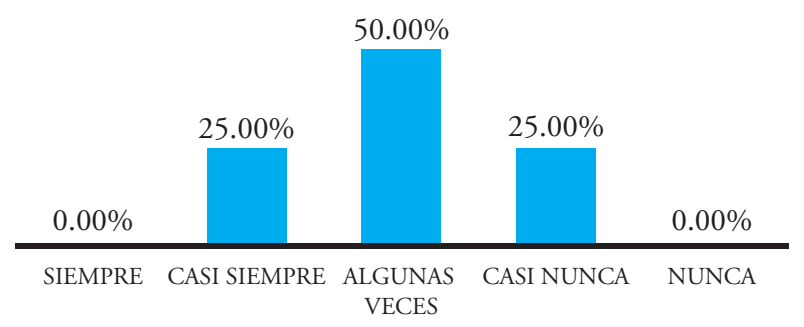

Frente a la pregunta “ ¿ $\mathrm{Ha}$ desmejorado el incumplimiento de las recomendaciones orientadas a mejorar la gestión de la entidad los procedimientos?",
$33,33 \%$, de los encuestados contestaron "casi siempre”; 33,33\%, "algunas veces"; 25\%, "casi nunca”; y $10 \%$, "nunca” (ver figura 3 ).

\section{Figura 3. Gráfico de distribución de frecuencia -} pregunta 3

3. ¿Ha desmejorado el incumplimiento de las recomendaciones orientadas a mejorar la gestión de la entidad los procedimientos?

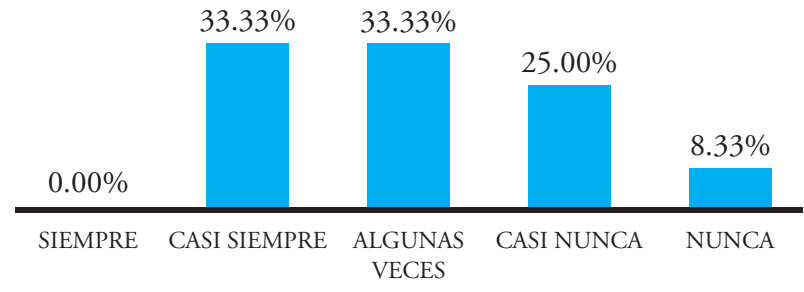

En relación con la interrogante “ ¿Ha desmejorado continuamente la implementación de acciones legales en la municipalidad por no tomar en cuenta las recomendaciones de los informes de auditoría?", $33,33 \%$ de los usuarios encuestados contestaron "casi siempre"; 33,33\%, "algunas veces"; 16,67\% "casi nunca"; 8,33\%, "nunca"; y 8,33\%, "siempre" (ver figura 4 ).

Figura 4. Gráfico de distribución de frecuencia pregunta 4

4. ¿¿Ha desmejorado continuamente la implementación de acciones legales en la municipalidad por no tomar en cuenta las recomendaciones de los informes de auditoría?

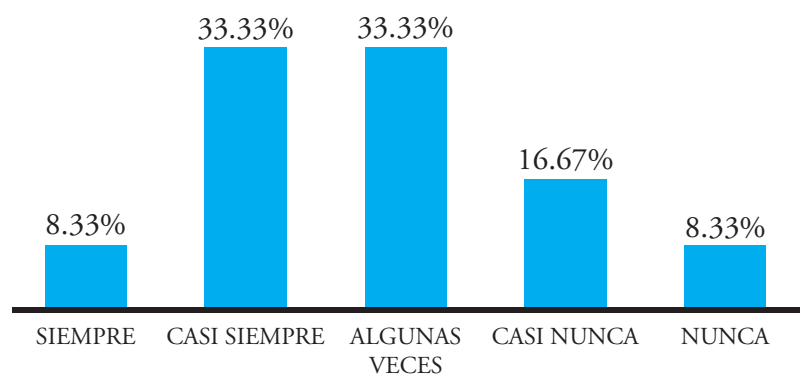


En el caso de la interrogante "¿Ha sido beneficiosa la eficiencia en el cumplimiento de las tareas?”, 41,67\% de los encuestados contestaron "algunas veces"; 41,67\%, "casi siempre”; y 16,67\%, "siempre” (ver figura 5).

Figura 5. Gráfico de distribución de frecuencia pregunta 5

5. ¿Ha sido beneficiosa laeficiencia en el cumplimiento de las tareas?

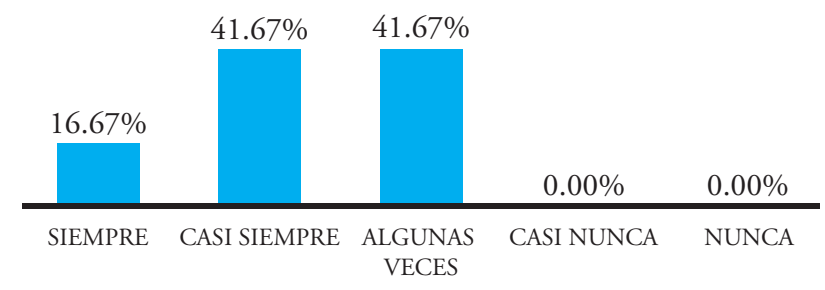

Con respecto a la interrogante "¿Se ha garantizado eficazmente el cumplimiento de los objetivos por la gestión de los gobiernos locales?”, 33,33\%, de los encuestados contestaron "algunas veces"; 33,33\%, "casi nunca"; 16,67\%, "casi siempre"; y 16,67\%, "casi nunca” (ver figura 6).

\section{Figura 6. Gráfico de distribución de frecuencia - pregunta 6}

6. ¿Se ha garantizado eficazmente el cumplimiento de los objetivos por la gestión de los gobiernos locales?

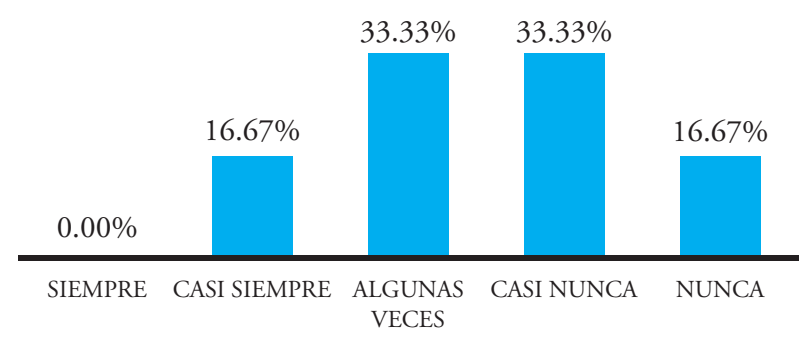

Por último, en la interrogante “ ¿Ha sido eficaz el nivel de satisfacción social para la municipalidad?”,
$58,33 \%$ de los encuestados contestaron "algunas veces"; 16,67\%, "casi siempre”; 16,67\%, "casi nunca”; y $8,33 \%$, "nunca" (ver figura 7 ).

\section{Figura 7. Gráfico de distribución de frecuencia -} pregunta 7

7. ¿Ha sido eficaz el nivel de satisfacción social para la municipalidad?

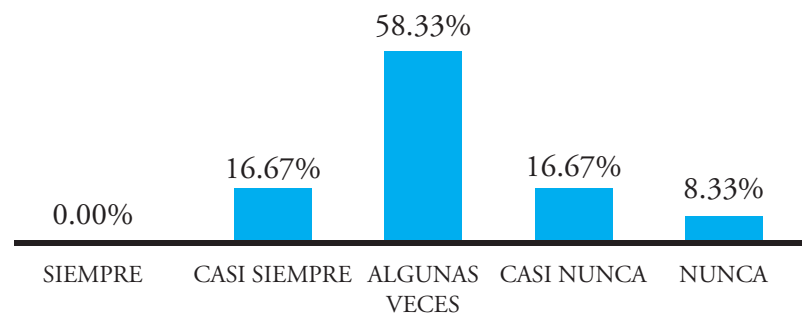

\section{Discusión}

Tras haber realizado los análisis correspondientes para medir la relación de la implementación de las recomendaciones de la auditoría y gestión de los gobiernos locales de Lima Metropolitana para el periodo 2015-2020, se pudo evidenciar el efecto que tiene la primera variable sobre el objeto de estudio. De esta manera, se han observado resultados similares a los de otras investigaciones, como los obtenidos por Gaibor (2015). En su investigación, se determina una conexión entre las recomendaciones de auditoría y su incidencia en los resultados de los procedimientos de administración y finanzas de gobiernos autónomos descentralizados municipales, los que presentan la misma situación de las municipalidades que integra Lima Metropolitana.

Siguiendo esa línea, Ramos (2017) evaluó el empleo de las sugerencias procedentes de dictámenes de auditoría en la gestión del Gobierno Regional de Puno y calificó de forma negativa los resultados de dicha ges- 
tión en el periodo 2013-2014. No obstante, la implementación de las recomendaciones de la auditoría y gestión de gobiernos locales de Lima Metropolitana para el periodo 2015-2020 ha sido medianamente aceptable de acuerdo a los usuarios encuestados en el presente estudio. Por su parte, Medrano (2017) evaluó las faltas del dictamen de auditoría financiera en los gobiernos locales de Lima Metropolitana. Así, observa una influencia positiva en la identificación de errores e irregularidades. Frente a este caso, en la presente investigación, el uso de tales recomendaciones es valorado de manera medianamente positiva.

Asimismo, Molina (2018) analizó la implementación de recomendaciones de los informes de auditoría de cumplimiento en la gestión administrativa de la Municipalidad Distrital de Acoria. Así, incluye una ponderación mínima de las acciones de control; en cambio, de acuerdo a la presente investigación, las recomendaciones de los informes de auditoría han contribuido significativamente en la productividad y el cumplimiento de las tareas. Por último, Apaico (2019) tuvo como fin determinar que la implementación de recomendaciones de los informes de auditorías gubernamentales incide en la gestión administrativa de la Municipalidad Provincial de Huamanga, y demostró que la implementación de las recomendaciones fue altamente significativa durante el año 2018. Frente a ello, en el presente estudio, la implementación de las recomendaciones incidió representativamente en la gestión de los gobiernos locales de Lima Metropolitana durante el periodo 2015-2020 por el cumplimiento de los objetivos, la satisfacción social y la entrega oportuna de los servicios a la comunidad.

Entre las conclusiones, se resalta que la eficiencia en el cumplimiento de las tareas de los gobiernos locales mencionados ha sido valorada de forma regular, principalmente, por la poca adecuación a las recomenda- ciones señaladas por el SNC. Además, la productividad en estas instituciones ha sido regularmente positiva cuando es vinculada a la capacidad de manejo de los recursos, aunque no haya sido considerada medianamente favorable en la gestión. El nivel de satisfacción social ha sido considerado regular puesto que la gestión de los gobiernos locales no ha sido tan favorable, principalmente, por no precisar adecuadamente acciones productivas como las que establecen las recomendaciones del SNC. La relación costo/beneficio en la dirección de los gobiernos organizados de forma local en los periodos 2015-2020 ha sido valorada como regular, dado que la falta del empleo adecuado de las medidas del SNC ha devenido en una economía de los recursos moderadamente estable en dichas entidades públicas. En líneas generales, el incumplimiento de los gobiernos locales de la muestra de 12 municipalidades ha sido regular en la implementación de las recomendaciones de auditoría del SNC.

Tal como lo señalan las Normas Generales de Control Gubernamental, el titular de la entidad es el responsable de instalar las acciones y escoger a los delegados de la implantación de las recomendaciones formuladas en los resultados de la ejecución de los servicios de control. De esta manera, se mantiene un proceso constante de evaluación de los avances logrados hasta obtener su total ejecución. Además, se debe notificar a la CGR y al OCI en la oportunidad y manera que instituya la Contraloría. De lo contrario, incurrirán en infracción sujeta a la potestad sancionadora de la Contraloría, de acuerdo con la normativa sobre la materia. Por tal motivo, se desprenden las siguientes recomendaciones:

- Emplear oportunamente las recomendaciones de las auditorias del SNC, principalmente, porque así se fomentará la eficiencia en el cumplimiento de las tareas y la gestión en los gobiernos locales para el bienestar social 
- Considerar tareas de aseguramiento de la calidad en el desarrollo de los procesos y cumplir con las normas que incrementen la productividad en el manejo de los recursos de las instituciones públicas

- Implementar las acciones del plan de acción propuesto, principalmente, porque, al fijar un convenimiento entre los gobiernos locales y las entidades auditoras del SNC, se va a fomentar un mayor compromiso en las municipales para hacer frente a los hallazgos detectados, además de incrementar el nivel de satisfacción social

- Emplear apropiadamente las medidas del SNC asociadas a la economía de los recursos asignados a las instituciones del sector público, buscando el equilibrio de la relación costo/beneficio en la gestión de los gobiernos locales

\section{Referencias bibliográficas}

Apaico, A. (2019). La implementación de las recomendaciones de los informes de auditoría gubernamentales y su efecto en la gestión administrativa de la Municipalidad Provincial de Huamanga, 2018 (Tesis de licenciatura, Facultad de Ciencias Contables, Financieras y Administrativas, Universidad Católica Los Ángeles de Chimbote. Chimbote, Perú). Recuperado de http://repositorio. uladech.edu.pe/bitstream/handle/123456789/11420/ AUDITORIA_GESTION_IMPLEMENTACION_RECOMENDACIONES_SEGUIMIENTO_APAICO_\%20PALOMINO_ARISTIDES. pdf? sequence $=1$ \&isAllowed=y f [Consulta: 8 de octubre de 2020].

Contraloría General de la Republica (2014, 13 de mayo). Normas Generales de Control Gubernamental [Resolución de Contraloría No 273-2014-CG]. Recuperado de https://portal.jne.gob.pe/portal_documentos/ files/5061a44b-0f1b-4205-9edb-c7b7a6edc3a6.pdf [Consulta: 8 de octubre de 2020].
Congreso de la República. (2003, 27 de mayo). Ley Orgánica de Municipalidades [Ley $\mathrm{N}^{\circ}$ 27923]. Recuperado de https://www2.congreso.gob.pe/sicr/cendocbib/ con4_uibd.nsf/BCD316201CA9CDCA0525810000 5DBE7A/\$FILE/1_2.Compendio-normativo-OT.pdf [Consulta: 8 de octubre de 2020].

Congreso de la República. (2018, 15 de mayo). Ley Orgánica del Sistema Nacional De Control y de la Contraloría General de la República [Ley No 27785]. Recuperado de https://www.oas.org/juridico/spanish/ mesicic2_ven_anexo_34_sp.pdf [Consulta: 8 de octubre de 2020].

Gaibor, M. (2015). Seguimiento de las recomendaciones de auditoría pública y su influencia en los resultados de los procesos administrativos y Financieros del Gobierno Autónomo Descentralizado Municipal del Cantón Montalvo de la Provincia de los Ríos (Tesis de licenciatura, Facultad de Ciencias Administrativas, Universidad de Guayaquil. Guayaquil, Ecuador. Recuperado de http:// repositorio.ug.edu.ec/bitstream/redug/14472/1/ TESIS \%20Cpa $\% 20140 \% 20$-\%20Seguimiento $\% 20 \mathrm{de} \% 20$ las $\% 20$ recomendaciones $\% 20 \mathrm{de} \% 20$ auditor\%c3\%ada\%20p\%c3\%bablica $\% 20$ y\% 20 su $\% 20$ influencia\%20en\%20los\%20resultados $\% 20$ de $\% 20$ los\%20procesos\%20administrativos $\% 20-\% 20$ MERCEDES\%20GAIBOR\% 20-\%20CPA $\% 20$ REVISI\%c3\%93N\%203.pdf [Consulta: 25 de octubre de 2016].

Instituto de Altos Estudios de Control Fiscal y Auditoría de Estado (COFAE). (2014). Informe de auditoría interna. Recuperado de http://www.oas.org/juridico/ PDFs/mesicic4_ven_inf_aud_int_2014.pdf [Consulta: 19 de octubre de 2016].

Instituto Nacional de Estadística e Informática (INEI). (2018). Sistemas de consulta. Población 2000 al 2015. Recuperado de http://proyectos.inei.gob.pe/web/ poblacion/ [Consulta: 19 de octubre de 2020].

Medrano, F. (2017). Evaluación de deficiencias del informe de auditoría financiera en gobiernos locales de Lima Metropolitana (Tesis de magíster, Facultad de Ciencias 
Contables-Unidad de Posgrado, Universidad Nacional Mayor de San Marcos. Lima, Perú). Recuperado de https://cybertesis.unmsm.edu.pe/bitstream/handle/20.500.12672/6153/Medrano_mf.pdf?sequence=1 [Consulta: 10 de febrero de 2019].

Ministerio de Economía y Finanzas (MEF). (2015, 6 de diciembre). Ley del presupuesto del sector público para el año fiscal 2016. Anexo 7 - Distribución del gasto público por gobiernos locales y fuentes de financiamiento Distribución del gasto del presupuesto del sector público por gobiernos locales y genéricos del gasto [Ley $\mathrm{N}^{\circ}$ 30372] Recuperado de https://www. mef.gob.pe/contenidos/presu_publ/sectr_publ/presu_2016/anexos/anexo_7.pdf [Consulta: 19 de octubre de 2016].

Molina, M. (2018). Análisis de la implementación de recomendaciones de los informes de auditoría de cumplimiento en la gestión administrativa de la Municipalidad Distrital de Acoria-Huancavelica: 2015-2016 (Tesis de licenciatura, Facultad de Ciencias Administrativas, Contables y Económicas, Universidad Nacional del Centro del Perú. Huancayo, Perú). Recuperado de http://repo-
sitorio.uncp.edu.pe/bitstream/handle/UNCP/4898/ Molina\%20Salas.pdf? sequence $=1$ \&isAllowed $=y$ [Consulta: 10 de febrero de 2019].

Ramos, A. (2017). Evaluación a la implementación de recomendaciones derivadas de informes de auditoría y su incidencia en la gestión del Gobierno Regional de Puno, periodos 2013-2014 (Tesis de licenciatura, Facultad de Ciencias Contables y Administrativas, Universidad Nacional del Altiplano. Puno, Perú). Recuperado de http://repositorio.unap.edu.pe/bitstream/ handle/UNAP/4775/Ramos_Ramos_Ana_Maria. pdf? sequence $=1$ \&isAllowed $=y$ [Consulta: 10 de febrero de 2019].

Wang, T., Liu, C., \& Chang, C.-H. (2011). CPA-firm merger: An investigation of audit quality'. European Accounting Review, 20(4), 727-761. https://doi.org/1 $0.1080 / 09638180.2011 .600485$

Fecha de recepción: 06 de noviembre de 2020 Fecha de aceptación: 24 de abril de 2021 Correspondencia: mmogrovejoe@unmsm.edu.pe 